\title{
Evaluation of the efficacy and safety of lanreotide in combination with targeted therapies in patients with neuroendocrine tumours in clinical practice: a retrospective cross-sectional analysis
}

Jaume Capdevila ${ }^{1 *}$, Isabel Sevilla², Vicente Alonso ${ }^{3}$, Luís Antón Aparicio ${ }^{4}$, Paula Jiménez Fonseca ${ }^{5}$, Enrique Grande ${ }^{6}$, Juan José Reina ${ }^{7}$, José Luís Manzano ${ }^{8}$, Juan Domingo Alonso Lájara ${ }^{9}$, Jorge Barriuso ${ }^{10}$, Daniel Castellano ${ }^{11}$, Javier Medina ${ }^{12}$, Carlos López ${ }^{13}$, Ángel Segura ${ }^{14}$, Sergio Carrera ${ }^{15}$, Guillermo Crespo ${ }^{16}$, José Fuster ${ }^{17}$, Javier Munarriz ${ }^{18}$ and Pilar García Alfonso ${ }^{19}$

\begin{abstract}
Background: Based on the mechanism of action, combining somatostatin analogues (SSAs) with mTOR inhibitors or antiangiogenic agents may provide synergistic effects for the treatment of patients with neuroendocrine tumours (NETs). Herein, we investigate the use of these treatment combinations in clinical practice.

Methods: This retrospective cross-sectional analysis of patients with NETs treated with the SSA lanreotide and targeted therapies at 35 Spanish hospitals evaluated the efficacy and safety of lanreotide treatment combinations in clinical practice. The data of 159 treatment combinations with lanreotide in 133 patients was retrospectively collected.

Results: Of the 133 patients, with a median age of 59.4 (16-83) years, 70 (52.6 \%) patients were male, 64 (48.1\%) had pancreatic NET, 23 (17.3\%) had ECOG PS $\geq 2,41$ (30.8 \%) had functioning tumours, 63 (47.7 \%) underwent surgery of the primary tumour, 45 (33.8 \%) had received prior chemotherapy, and 115 (86.5 \%) had received prior SSAs.

115 patients received 1 lanreotide treatment combination and 18 patients received between 2 and 5 combinations. Lanreotide was mainly administered in combination with everolimus (73 combinations) or sunitinib (61 combinations). The probability of being progression-free was $78.5 \%$ (6 months), $68.6 \%$ (12 months) and $57.0 \%$ (18 months) for patients who only received everolimus plus lanreotide $(n=57)$ and $89.3 \%$ (6 months), $73.0 \%$ (12 months), and $67.4 \%$ (18 months) for patients who only received sunitinib and lanreotide $(n=50)$. In patients who only received everolimus plus lanreotide the median time-to-progression from the initiation of lanreotide combination treatment was 25.8 months $(95 \% \mathrm{Cl}, 11.3,40.3)$ and it had not yet been reached among the subgroup of patients only receiving sunitinib plus lanreotide. The safety profile of the combination treatment was comparable to that of the targeted agent alone.
\end{abstract}

Conclusions: The combination of lanreotide and targeted therapies, mainly everolimus and sunitinib, is widely used in clinical practice without unexpected toxicities and suggests efficacy that should be explored in randomized prospective clinical trials.

Keywords: Lanreotide, Neuroendocrine tumours, Sunitinib, Everolimus, Somatostatin analogues, Clinical practice, Cross-sectional analysis, Combination treatment

\footnotetext{
* Correspondence: jacapdevila@vhebron.net

${ }^{1}$ Medical Oncology Department, Vall d'Hebron University Hospital, Autonomous University of Barcelona, P. Vall d'Hebron 119-129, 08035 Barcelona, Spain

Full list of author information is available at the end of the article
}

\section{Biomed Central}

(C) 2015 Capdevila et al. This is an Open Access article distributed under the terms of the Creative Commons Attribution License (http://creativecommons.org/licenses/by/4.0), which permits unrestricted use, distribution, and reproduction in any medium, provided the original work is properly credited. The Creative Commons Public Domain Dedication waiver (http:// creativecommons.org/publicdomain/zero/1.0/) applies to the data made available in this article, unless otherwise stated. 


\section{Background}

Neuroendocrine tumours (NETs) are a heterogeneous group of relatively rare malignancies originating from the diffuse neuroendocrine system found most often in the bronchial or gastrointestinal systems [1]. Somatostatin analogues (SSAs) are a key therapeutic option in the management of advanced NETs, leading to a significant improvement in patient quality of life [2-5]. There are currently 2 SSAs in clinical use: octreotide [6] and lanreotide $[7,8]$. Longer acting (slow-release and depot) formulations of SSAs include octreotide long-acting release (LAR), lanreotide Autogel and lanreotide LP. Small studies have suggested that treatment with SSAs is associated with disease stabilization and prolonged progression-free survival (PFS) in some patients with NETs [8, 9]. Moreover, following the randomized PROMID study confirming that octreotide delayed time to tumour progression (TTP) (from 6 to 14.3 months, hazard ratio $[\mathrm{HR}]=0.34 ; \mathrm{p} \leq$ 0.0001) in patients with metastatic NETs [9], SSAs have been administered to patients to provide not only hormonal symptom control but also antitumour activity [10].

A Phase II trial carried out by the Spanish TTD group evaluated the efficacy of lanreotide Autogel $120 \mathrm{mg}$ on tumour growth stabilization in 30 patients with progressive gastroenteropancreatic and bronchopulmonary NETs. The median PFS was 12.9 months with clinical benefit reported in $93 \%$ of the patients [11]. In the international Phase III Clarinet trial lanreotide substantially prolonged PFS compared with placebo ( $\mathrm{HR}=0.47 ; 95 \%$ CI 0.30 $0.73 ; \mathrm{p}<0.001)$ in patients with non-functioning gastroenteropancreatic NETs [12].

Recent therapeutic advances with everolimus, a mammalian target of rapamycin (mTOR) inhibitor, and sunitinib, a multitargeted agent with antiangiogenic activity, have led to an improvement in patients with advanced pancreatic NETs (pNETs) [13-16]. Everolimus has shown antitumour activity in 2 Phase III studies (RADIANT-2 and RADIANT-3). In RADIANT-2, treatment with everolimus plus octreotide resulted in a 5.1-month increase in median PFS compared with placebo plus octreotide (16.4 vs. 11.3 months) in patients with advanced NETs with carcinoid syndrome, although the difference did not reach statistical significance [13]. In RADIANT-3, patients with progressive pNETs had a statistically significant improvement in PFS associated with everolimus compared with placebo (11 vs. 4.6 months). A Phase III study of sunitinib in patients with progressive pNETs was unblinded early after more than a doubling of median PFS (11.4 vs. 5.5 months) favoured the patients receiving sunitinib vs. placebo [14]. After a 2-year follow-up, the median overall survival (OS) was estimated at 33 months in the sunitinib arm [17].

The combination of SSAs and targeted therapies is a potential treatment option for patients with NETs [18].
Indeed, several small studies suggest that the combined use of octreotide and everolimus could provide an increase in efficacy $[13,19,20]$. Unfortunately, no randomized data have compared the outcome of patients who received a novel targeted agent alone $v s$. the combination with a SSA. However, in clinical practice, targeted therapies are frequently combined with SSAs and there have been reports of valuable efficacy in heavily pretreated patients [21]; thus in a retrospective cohort, $83 \%$ of 29 patients with well differentiated pNETs who were treated with sunitinib in daily practice in Spain also received concomitant treatment with SSAs [22]. Furthermore, Barriuso et al., reported that $87.5 \%$ of 40 patients with NETs on treatment with sunitinib as palliative treatment in 6 Spanish hospitals, concomitantly received SSAs [23].

The aim of this retrospective cross-sectional analysis was to define the efficacy and safety of the SSA, lanreotide, in combination with antiangiogenic targeted therapies or inhibitors of the mTOR pathway in the routine clinical practice, to help evaluate their potential clinical benefit in the management of patients with NETs in Spain.

\section{Methods \\ Design}

Between July 2011 and October 2011 we collected the data from patient medical charts to perform a retrospective multicentre cross-sectional analysis of patients with NETs that were treated with the SSA lanreotide combined with novel targeted therapies. Data were collected from medical oncology services of Spanish hospitals with experience in the treatment of NETs with lanreotide and newer therapeutic agents, such as mTOR inhibitors or antiangiogenic agents (tyrosine kinase inhibitors [TKIs] or monoclonal antibodies). Thirty-five centres distributed over 27 Spanish provinces were identified and invited to participate in the project. The conduct of this retrospective cross-sectional analysis was approved by the ethics committee of the Vall d'Hebron University Hospital.

\section{Objectives}

We wanted to determine the epidemiologic characteristics of the patients analysed, in terms of proliferative rate and location of the primary tumour, functionality, differentiation and tumour extension, as well as treatments received prior to the combination therapy. The main efficacy objectives included determining the drugs used in the course of the combined therapy, the length of this combination therapy, biochemical response (50\% reduction of chromogranin A), the radiologic response rate obtained according to Response Evaluation Criteria In Solid Tumours (RECIST) v1.0, and response duration. The radiologic images were not centrally reviewed by the investigators; the information on progression was 
Table 1 Patient demographics, disease characteristics, and prior treatment regimens

\begin{tabular}{ll}
\hline Characteristic & Number of patients \\
& $(\mathrm{N}=133)$
\end{tabular}

Sex, n (\%)

Male

$70(52.6)$

Female

$63(47.4)$

Age, years

Median (range)

$59.4(16-83)$

Comorbidities, n (\%)

Hypertension

$51(38.3)$

Diabetes

Dyslipidaemia

$37(27.8)$

25 (18.8)

Heart disease

19 (14.3)

Liver disease

Hypothyroidism

Tumour extension at diagnosis, $\mathrm{n}(\%)$

Locally advanced

Metastatic

Tumour extension at treatment initiation, $\mathrm{n}(\%)$

Locally advanced

$2(1.5)$

Metastatic

ECOG PS, n (\%)

0

1

2

3

Location of primary tumour, $\mathrm{n}(\%)$

$\begin{array}{ll}\text { Foregut } & 85(64.0) \\ \text { Lung } & 12(9.0) \\ \text { Oesophagus } & 1(0.8) \\ \text { Stomach } & 3(2.3) \\ \text { Duodenum } & 5(3.8) \\ \text { Pancreas } & 64(48.1) \\ \text { Midgut } & 30(22.6) \\ \text { Jejunum } & 3(2.3) \\ \text { lleum } & 21(15.8) \\ \text { Appendix } & 2(1.5) \\ \text { Cecum } & 4(3.0) \\ \text { Hindgut } & 6(4.5) \\ \text { Colon } & 3(2.3) \\ \text { Rectum } & 3(2.3) \\ \text { Unknown } & 12(9.0) \\ \text { Histological differentiation, n (\%) } & \\ \text { Grade 1 } & 55(41.4) \\ \text { Grade 2 } & 42(31.6)\end{array}$

Table 1 Patient demographics, disease characteristics, and prior treatment regimens (Continued)

\begin{tabular}{|c|c|}
\hline Grade 3 & $2(1.5)$ \\
\hline Unknown & $34(25.6)$ \\
\hline \multicolumn{2}{|l|}{ Location of metastases, n (\%) } \\
\hline Liver & $112(84.2)$ \\
\hline Bone & $18(13.5)$ \\
\hline Peritoneum & $19(14.3)$ \\
\hline Lung & $19(14.3)$ \\
\hline Lymph node & $29(21.8)$ \\
\hline Other $^{a}$ & $4(3.0)$ \\
\hline \multicolumn{2}{|l|}{ Tumour functionality, n (\%) } \\
\hline Non-functioning & $92(69.2)$ \\
\hline Functioning & $41(30.8)$ \\
\hline Carcinoid & $13(9.8)$ \\
\hline Gastrinoma & $1(0.8)$ \\
\hline Somatostinoma & $1(0.8)$ \\
\hline VIPoma & $3(2.3)$ \\
\hline Not specified & $23(17.3)$ \\
\hline \multicolumn{2}{|l|}{ Ki-67 index, n (\%) } \\
\hline $0-2$ & $52(39.1)$ \\
\hline $3-10$ & $29(21.8)$ \\
\hline $11-20$ & $8(6.0)$ \\
\hline$>20$ & $1(0.8)$ \\
\hline Unknown & $43(32.3)$ \\
\hline \multicolumn{2}{|c|}{ Previous non-pharmacologic treatments, n (\%) } \\
\hline Surgery of the primary tumour & $63(47.4 \%)$ \\
\hline Surgery of metastases & $21(15.8 \%)$ \\
\hline Local treatments ${ }^{\mathrm{b}}$ & $23(17.3)$ \\
\hline Radiotherapy & $6(4.5)$ \\
\hline \multicolumn{2}{|c|}{ Previous pharmacologic treatments, n (\%) } \\
\hline None & $16(12.0)$ \\
\hline Chemotherapy & $45(33.8)$ \\
\hline Targeted therapy & $16(12.0)$ \\
\hline Immunotherapy & $7(5.3)$ \\
\hline \multicolumn{2}{|l|}{ Somatostatin analogues } \\
\hline Lanreotide & $78(58.6)$ \\
\hline Octreotide & $37(27.8)$ \\
\hline \multicolumn{2}{|c|}{ Combination with somatostatin analogues } \\
\hline Interferon & $19(14.3)$ \\
\hline Targeted therapy & $7(5.3)$ \\
\hline
\end{tabular}

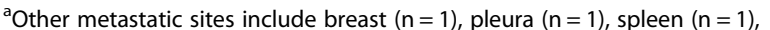
adrenal gland $(n=1)$

bIncludes embolization, (transarterial) chemoembolization, radiofrequency ablation and radioembolization

ECOG PS, Eastern Cooperative Group Oncology Performance Status; VIPoma, Vasoactive intestinal peptide secreting tumour 
obtained from the patients' medical chart. TTP was defined as the time from the initiation of lanreotide combination therapy until there was an indication of disease progression as noted in the patients' clinical history. In line with the retrospective nature of this analysis, it is important to point out that the progression status had no planning dates for the estimation of TPP. OS was defined from the initiation of lanreotide combination therapy until patient death. Safety objectives were to collect the reasons for discontinuing the combined therapy, and to define the adverse events (AEs) profile according to the Common Terminology Criteria for Adverse Events (CTCAE) v3.0.

\section{Patient population}

All patients diagnosed with NET being followed at the medical oncology services who had received treatment with lanreotide in combination with a novel therapeutic target agent for at least 3 months prior to data collection into an electronic Data Report Form were eligible to be included in the retrospective analysis. All patients had progressed on previous treatment before receiving combination treatment with lanreotide. If the length of combination treatment did not reach 3 months, the patient would still be eligible for inclusion as long as treatment discontinuation was due to an AE. The 3-month minimum combined treatment cut-off would be used to exclude patients who abandoned combination treatment very early; however, there were patients included in the analysis who received combined treatment for less than 3 months. Upon progression with the lanreotide combination, patients received further treatment according to the standard of care at each centre.

\section{Statistical analysis}

Summary statistics are presented for all variables. Efficacy was assessed on the basis of tumour response. Kaplan-Meier methods were used to obtain estimates of median TTP and OS, with corresponding HRs and 2-sided $95 \%$ confidence intervals (CIs). The protocol was approved by the Ethics Committee of the hospitals where data was collected.

\section{Results}

\section{Patient population}

One hundred and thirty-three patients with a diagnosis of NET who received combination treatment with lanreotide and targeted therapy in the setting of routine clinical practice were analysed. Patients began receiving lanreotide combination treatment between April 2008 and July 2011. The demographic and clinical characteristics of the patients are described in Table 1. The median age of patients with NETs was 59.4 years, and their main comorbidities were hypertension and diabetes. Approximately half of the patients had pNETs; the primary site was the ileum in 21 (15.8\%) patients and the lung in $12(9.0 \%)$ patients. Almost all patients had metastatic disease at diagnosis $(98.5 \%)$ and the liver was the most common metastatic location (84.2 \%). Thirty-one percent of patients had functional tumours (carcinoid, gastrinoma, somatostinoma and vasoactive intestinal peptide secreting tumour [VIP] oma). The majority of patients had received prior pharmacologic treatment. The number of prior treatment lines was 1 for $52(39.1 \%)$ patients, 2 for 31 (23.3\%) patients, 3 for 19 (14.3\%) patients, 4 for 9 (6.8 \%) patients, and 5 for 6 (4.5\%) patients.

\section{Treatment and patient disposition}

According to the investigators' criteria, the main reason for combining lanreotide with targeted therapies was to achieve antiproliferative synergy (113 patients, $85.0 \%$ ). In the other patients, the main reason was to control hormonal symptoms. The majority (115 patients, $86.5 \%$ ) of patients received only 1 lanreotide treatment combination; but, overall, the 133 patients included in the analysis received a total of 159 combinations of targeted therapy with lanreotide (Table 2) so there were patients that

Table 2 Treatment combinations in the 133 patients analysed

\begin{tabular}{ll}
\hline & $\begin{array}{l}\text { Number of patients } \\
(\mathbf{N}=133)\end{array}$ \\
\hline Number of treatment combinations, $\mathrm{n}(\%)$ & \\
1 & $115(86.0)$ \\
2 & $12(9.0)$ \\
3 & $5(3.8)$ \\
5 & $1(0.8)$
\end{tabular}

\section{Number of treatment} combinations

$(\mathrm{N}=159)$

Targeted agent combined with lanreotide, $\mathrm{n}(\%)$

$\begin{array}{cl}\text { Everolimus } & 73(45.9) \\ \text { Sunitinib } & 61(38.4) \\ \text { Treatment discontinuation, } \mathrm{n}(\%)^{\mathrm{a}} & 84(52.8) \\ \text { Everolimus } & \mathrm{n}=73 \\ \text { All discontinuations } & 39(53.4) \\ \text { Disease progression } & 23(31.5) \\ \text { Adverse event } & 10(13.7) \\ \text { Other } & 6(8.2) \\ \text { Ongoing } & 34(46.6) \\ \text { Sunitinib } & \mathrm{n}=61 \\ \text { All discontinuations } & 27(44.3) \\ \text { Disease progression } & 15(24.6) \\ \text { Adverse event } & 10(16.4) \\ \text { Other } & 2(3.3) \\ \text { Ongoing } & 34(55.7)\end{array}$

${ }^{a}$ The denominator is the number of treatment combinations with a each targeted agent 
received 2 or more combinations. As expected, the most common combinations were with everolimus (73 combinations, $45.9 \%$ of the 159 combinations) and sunitinib (61 combinations, $38.4 \%$ of the 159 combinations). Other combinations with targeted agents included bevacizumab $(\mathrm{n}=9)$, sorafenib $(\mathrm{n}=8)$, and pazopanib $(\mathrm{n}=8)$; however, due to the small number of patients that received each of these combinations individual characterization of the outcomes of these combinations was not carried out. With a median follow-up of 43.9 months (range 1.8-628.7), the median duration of treatment was 5.1 months (range $0-35.6)$ in the 115 patients who only received 1 treatment combination. In the 57 patients who only received everolimus plus lanreotide the median follow-up was 42.2 months (range 1.8-275.1) and the median duration of treatment was 4.7 months (range 0-35.6). Similarly, in the 50 patients who only received the combination of sunitinib with lanreotide, the median follow-up was 31.8 months (range 2.8-628.7), with a median duration of treatment of 5.9 months (range 0.4-25.0). There were 4 patients receiving everolimus plus lanreotide (range 0.59-2.95 months) and 2 patients receiving sunitinib plus lanreotide (range 0.39-2.98 months) that received treatment for less than 3 months and discontinued due to an AE. In addition there were 5 patients receiving the everolimus and lanreotide combination for less than 3 months that discontinued due to tumour progression (range 0-2.98 months).

In 128 of the 159 combinations the dose of lanreotide Autogel was $120 \mathrm{mg}$ every 28 days. Everolimus was administered at a dose of $10 \mathrm{mg} /$ day in 72 combinations and at $5 \mathrm{mg} /$ day in 1 combination. The administration of sunitinib was less homogeneous, 49 combinations with a continuous dose of $37.5 \mathrm{mg} /$ day, 11 combinations of $50 \mathrm{mg} /$ day sunitinib on a 4 weeks on/2 weeks off schedule, and 1 patient who received $25 \mathrm{mg} /$ day.

At the time of the data cut-off, 84 treatment combinations (52.8\% of 159) had been discontinued. The reasons for treatment discontinuation were disease progression in 47 (29.6\% of 159) combinations and AEs in 24 (15.1\% of 159 ) combinations (Table 2 ).

Data on follow-up treatment was collected for $30 \mathrm{pa}-$ tients. Fourteen patients received a SSA, either as monotherapy or in combination with another agent. Five patients received sunitinib, either as monotherapy or in combination with a SSA and four patients received everolimus, either as monotherapy or in combination with lanreotide. Eight patients received chemotherapy combinations.

\section{Efficacy}

Overall, 23 treatment combinations led to a tumour response (1 complete response [CR] and 22 partial responses [PRs]) resulting in an objective response rate of $14.5 \%$ with all treatment combinations (Table 3). Stable disease (SD) was reported in $113(71.1 \%)$ treatment combinations and the disease control rate was $85.5 \%$. The response (18.3\%; with $1 \mathrm{CR}$ and $20 \mathrm{PRs}$ ) and disease control rates $(82.6 \%)$ were similar in the subgroup of the 115 patients who only received 1 treatment combination. Chromogranin A expression was measured in 37 (27.8\%) patients, with normalization reported in 6 (16.2 \% of 37) patients and a reduction in 10 (27.0\% of 37) patients. A correlation between chromogranin A expression and

Table 3 Radiologic response rate in all 133 patients (analysed all 159 treatment combinations) and in the 115 patients that only received one lanreotide combination

\begin{tabular}{|c|c|c|c|}
\hline All patients & All treatment combinations & Everolimus + lanreotide & Sunitinib + lanreotide \\
\hline & $\mathrm{N}=159^{\mathrm{a}}$ & $\mathrm{n}=73$ & $n=61$ \\
\hline \multicolumn{4}{|l|}{ Tumour response (\%) } \\
\hline Complete response & $1(0.6)$ & 0 & $1(1.6)$ \\
\hline Partial response & $22(13.8)$ & $11(15.1)$ & $9(14.8)$ \\
\hline Stable disease & $113(71.1)$ & $49(67.0)$ & $42(68.9)$ \\
\hline Progressive disease & $13(8.2)$ & $9(12.3)$ & $3(4.9)$ \\
\hline Not evaluated & $10(6.2)$ & $4(5.5)$ & $6(9.8)$ \\
\hline \multirow[t]{2}{*}{ Patients that received only one lanreotide combination } & One treatment combination & & \\
\hline & $n=115$ & $\mathrm{n}=\mathbf{5 7}$ & $\mathrm{n}=50$ \\
\hline \multicolumn{4}{|l|}{ Tumour response (\%) } \\
\hline Complete response & $1(0.9)$ & 0 & $1(2.0)$ \\
\hline Partial response & $20(17.4)$ & $10(17.5)$ & $8(16)$ \\
\hline Stable disease & $74(64.3)$ & $35(61.5)$ & $34(68.0)$ \\
\hline Progressive disease & $11(9.6)$ & $8(14.0)$ & $2(4.0)$ \\
\hline Not evaluated & $9(7.8)$ & $4(7.0)$ & $5(10)$ \\
\hline
\end{tabular}

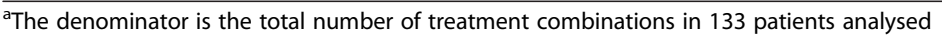


radiologic tumour response was not carried out because many chromogranin A measurements were missing. One third of the patients did not have Ki67 data and proliferation index was not analysed.
Subanalysis of patients that only received everolimus and lanreotide or sunitinib and lanreotide

Among the 115 patients who received only 1 lanreotide treatment combination, 57 patients received everolimus

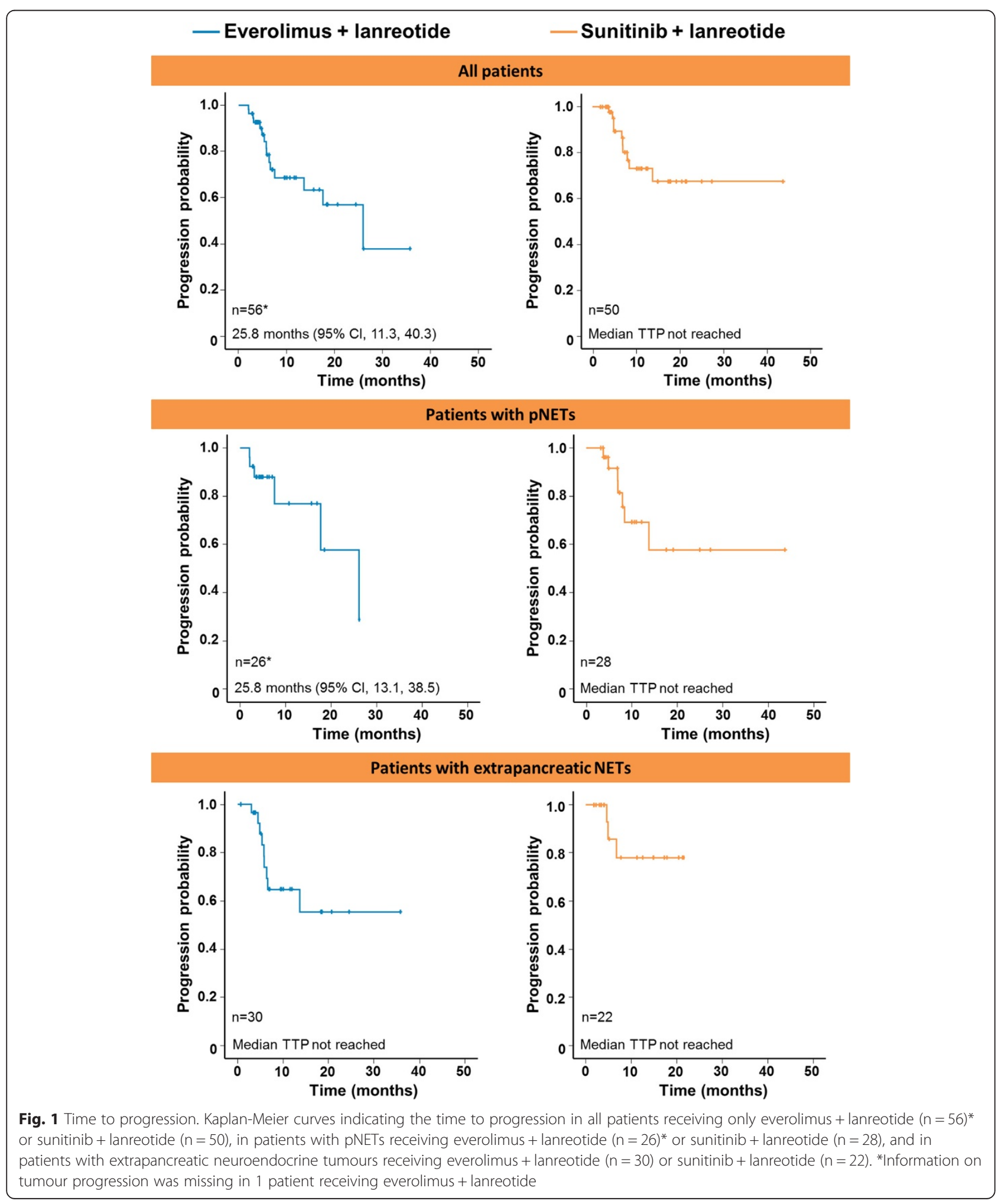


plus lanreotide and 50 patients received sunitinib plus lanreotide. In patients who only received everolimus plus lanreotide the median TTP from the initiation of combination treatment was 25.8 months (95\% CI, 11.3, 40.3) and it had not yet been reached among the subgroup of patients receiving sunitinib and lanreotide (Fig. 1). The probability of being progression-free at 6 months was $78.5 \%$ in the everolimus and lanreotide group and $89.3 \%$ in the sunitinib and lanreotide group and at 12 months it was 68.6 and $73.0 \%$, in the everolimus and sunitinib patient groups, respectively. At 18 months, $57.0 \%$ of patients receiving everolimus plus lanreotide and $67.4 \%$ of patients receiving sunitinib plus lanreotide were estimated to be free of progression. The median OS was 26.4 months (95 \% CI, 17.5, 35.4) for patients receiving everolimus and lanreotide and 32.8 months (95\% CI, 12.5, 53.0) for sunitinib subgroup (Fig. 2).

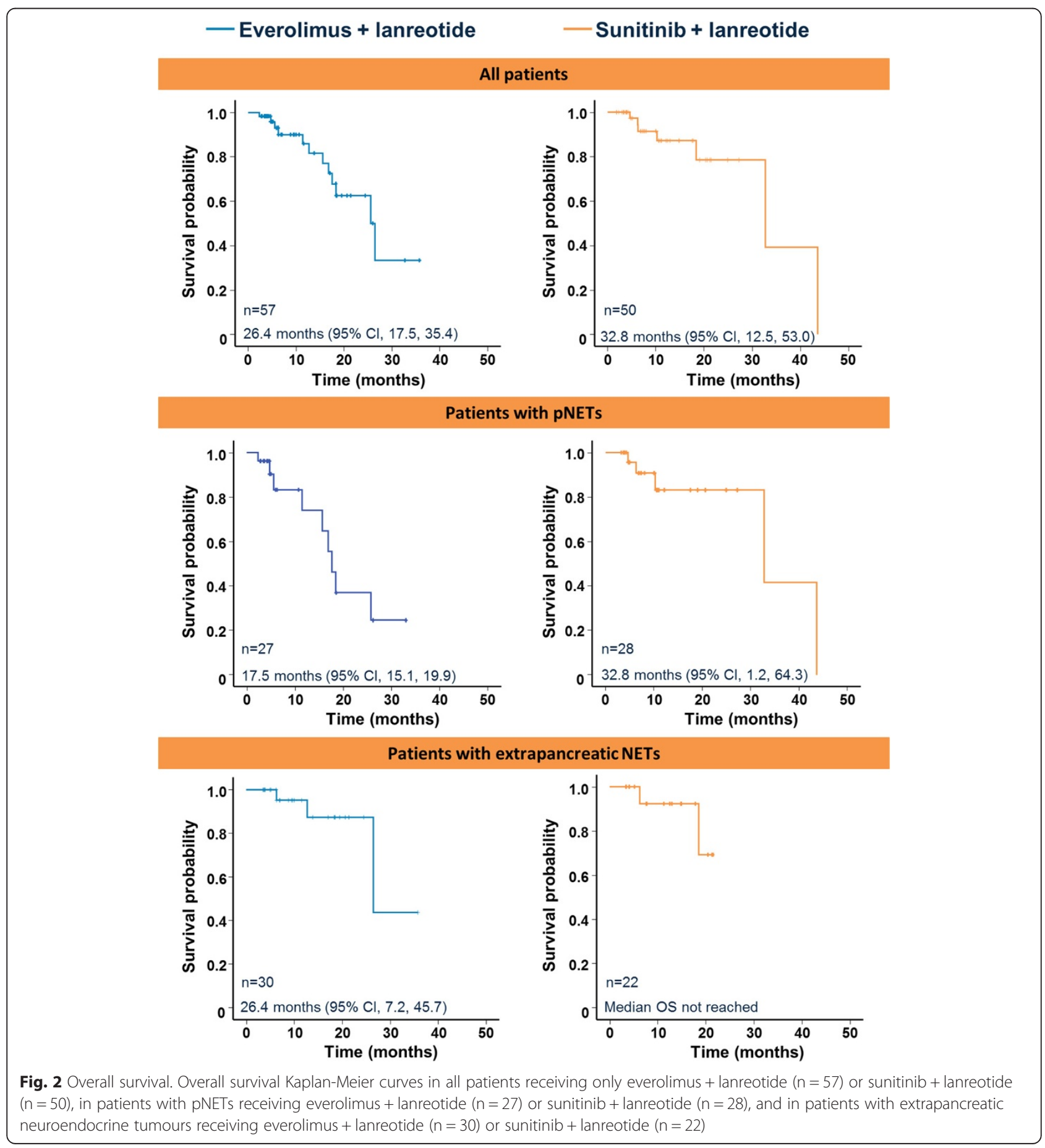




\section{Safety}

Overall there were 270 AEs in 97 patients (Table 4). The majority of AEs reported were Grade $1(\mathrm{n}=115)$ or $2(\mathrm{n}=106)$ in severity, with few Grade $3(\mathrm{n}=39)$ or Grade $4(n=9)$ AEs. Generally, the safety profile of the combination with lanreotide resembled the safety profile of the targeted agent in monotherapy (Table 5). The main AEs were asthenia, mucositis, and diarrhoea. There were 6 AEs (5 AEs were Grade 1 or 2) that were related to lanreotide administration; these included diarrhoea, hyperglycaemia, and abdominal pain.

At the data cut-off, 3 patients were alive without disease, 106 patients were alive with disease and there had been 24 deaths (22 due to disease progression, 1 cardiac insufficiency and 1 death in a patient receiving everolimus plus lanreotide that was not due to disease progression and was potentially considered by the investigator to be a Grade $5 \mathrm{AE}$ ).

Table 4 Treatment-related AEs in all patients; $N=133$

\begin{tabular}{lll}
\hline & All AEs & Grade $\geq 3$ \\
\hline Asthenia & $48(36.1)$ & $\mathrm{n}(\%)$ \\
Mucositis & $35(26.3)$ & $13(9.8)$ \\
Diarrhoea & $33(24.8)$ & $6(4.5)$ \\
Hand-foot skin reaction & $20(15.0)$ & $4(3.0)$ \\
Anorexia & $16(12.0)$ & $1(0.8)$ \\
Hyperglycaemia & $13(9.8)$ & $2(1.5)$ \\
Rash & $12(9.0)$ & $1(0.8)$ \\
Hypertension & $12(9.0)$ & $3(2.3)$ \\
Peripheral oedema & $7(5.3)$ & $1(0.8)$ \\
Thrombocytopenia & $7(5.3)$ & $2(1.5)$ \\
Anaemia & $7(5.3)$ & $3(2.3)$ \\
Pneumonitis & $4(3.0)$ & $1(0.8)$ \\
Cardiac toxicity & $4(3.0)$ & $1(0.8)$ \\
Hypercholesterolemia & $4(3.0)$ & $3(2.3)$ \\
Hypertriglyceridemia & $4(3.0)$ & 0 \\
Leucopoenia & $4(3.0)$ & 0 \\
Hepatic alterations & $3(2.3)$ & $1(0.8)$ \\
Hypothyroidism & $3(2.3)$ & 0 \\
Nausea & $3(2.3)$ & 0 \\
Vomiting & $3(2.3)$ & 0 \\
Abdominal pain & $3(2.3)$ & 0 \\
Weight loss & $3(2.3)$ & 0 \\
Headache & $1(0.8)$ & 0 \\
Epistaxis & $1(0.8)$ & 0 \\
Other & $20(15.0)$ & 0 \\
\hline AE & & 0 \\
\hline & & 0 \\
\hline
\end{tabular}

$\mathrm{AE}=$ adverse event

\section{Discussion}

This cross-sectional analysis retrospectively evaluated the clinical use of the SSA lanreotide in combination with targeted agents in Spanish patients with advanced NETs in the setting of routine clinical practice. As expected, in the majority of patients, lanreotide was administered with everolimus or sunitinib. The probability of being progression-free was encouraging in the patient population analysed (patients who survived or maintained treatment for more than 3 months). The estimated proportion of patients who were alive and progression-free at 18 months was $34 \%$ with everolimus in the RADIANT-3 trial [15] and in the sunitinib Phase 3 trial it was estimated that $71.3 \%$ of patients were alive and progression-free at 6 months [14].

In the RADIANT- 3 trial there were $40 \%$ of patients that received concomitant treatment with SSAs, but median PFS for treatment with everolimus was similar in the group of patients that received SSAs (11.4 months) and in the group of patients that did not (10.8 months) [24]. In the Phase II RADIANT-1 study, the median PFS by central radiology review was 16.7 months and the median OS had not been reached at the time of data cut-off in the subgroup of patients who received everolimus plus octreotide [19]. In the subgroup of patients receiving everolimus monotherapy median PFS was 9.7 months and median OS was 24.9 months. A subanalysis of the $40 \%$ of patients receiving SSAs in the Phase III sunitinib study showed that their use resulted in a nonstatistically significant improvement in PFS (HR 0.78; p = 0.31) compared with the patients who received no on-study SSA [25].

In our cross-sectional analysis there might appear to be differences in the efficacy results between everolimus or sunitinib; however, this analysis was not set up to compare the data between the different targeted agents that are routinely combined with lanreotide in clinical practice and therefore it should not be assumed that one of the targeted agents analysed here would be a better combination partner for lanreotide. There are several limitations that should be taken into account when dissecting the data in our cohort of patients. This is a cross-sectional and retrospective analysis of patients being treated at selected sites that are presumed to be reference sites for the treatment of NETs and to have experience in the management of novel targeted agents. Furthermore, there was a bias in the selection process since the patients included in this retrospective analysis should have been receiving treatment for at least 3 months except for those who did not tolerate the combination. This inherent selection bias probably underestimates the number of patients in clinical practice with early progression with the combination strategy. There were no strict timelines to assess tumour response, no central review of the images, and patient follow up was 
Table 5 Number of adverse events (AEs) and Grade 3 or 4 AEs reported during the study and assignment of causality to the treatment received. The number of Grade 3 and 4 AEs is shown in parenthesis

\begin{tabular}{|c|c|c|c|c|c|}
\hline & Everolimus & Lanreotide and everolimus & Sunitinib & Lanreotide and sunitinib & Lanreotide \\
\hline & All AEs (Grade 3-4) & All AEs (Grade 3-4) & All AEs (Grade 3-4) & All AEs (Grade 3-4) & All AEs (Grade 3-4) \\
\hline$A E$ & $129(21)$ & $9(2)$ & $70(17)$ & $15(5)$ & $6(1)$ \\
\hline Asthenia & $15(5)$ & 0 & $22(8)$ & $2(0)$ & 0 \\
\hline Mucositis & $25(6)$ & 0 & $6(0)$ & 0 & 0 \\
\hline Diarrhoea & $17(2)$ & $1(0)$ & $5(1)$ & $5(1)$ & $1(0)$ \\
\hline Hand-foot skin reaction & $8(0)$ & 0 & $8(1)$ & 0 & 0 \\
\hline Anorexia & $9(1)$ & 0 & $4(1)$ & 0 & 0 \\
\hline Hyperglycaemia & $7(0)$ & $4(1)$ & 0 & $1(0)$ & $1(0)$ \\
\hline Rash & $10(2)$ & $1(1)$ & 0 & 0 & 0 \\
\hline Hypertension & $2(0)$ & 0 & $6(1)$ & 0 & 0 \\
\hline Peripheral oedema & $6(2)$ & 0 & $1(0)$ & 0 & 0 \\
\hline Thrombocytopenia & 0 & 0 & $4(0)$ & $3(3)$ & 0 \\
\hline Anaemia & $5(0)$ & 0 & 0 & $2(1)$ & 0 \\
\hline Pneumonitis & $3(1)$ & 0 & $1(0)$ & 0 & 0 \\
\hline Cardiac toxicity & 0 & 0 & $3(2)$ & 0 & 0 \\
\hline Hypercholesterolemia & $4(0)$ & 0 & 0 & 0 & 0 \\
\hline Hypertriglyceridemia & $4(0)$ & 0 & 0 & 0 & 0 \\
\hline Leucopoenia & $1(0)$ & 0 & $2(1)$ & $1(0)$ & 0 \\
\hline Hepatic alterations & 0 & $2(0)$ & 0 & 0 & 0 \\
\hline Hypothyroidism & 0 & 0 & $3(0)$ & 0 & 0 \\
\hline Nausea & $1(0)$ & 0 & 0 & 0 & 0 \\
\hline Vomiting & $1(0)$ & 0 & $1(0)$ & 0 & 0 \\
\hline Abdominal pain & $1(0)$ & 0 & 0 & 0 & $2(0)$ \\
\hline Weight loss & $1(0)$ & 0 & 0 & $1(0)$ & 0 \\
\hline Headache & 0 & 0 & 0 & 0 & 0 \\
\hline Epistaxis & 0 & 0 & $1(0)$ & 0 & 0 \\
\hline Other & $9(2)$ & $1(0)$ & $3(2)$ & 0 & $2(1)$ \\
\hline
\end{tabular}

performed according to local guidelines. In addition, the sample of the analysis is very heterogeneous since there are several patients who received subsequent lines of treatment, including maintenance with lanreotide alone. Furthermore, the dose of sunitinib that patients received was heterogeneous; the majority of patients received continuous daily dosing (the schedule that is approved in Europe for patients with pNETs), but a considerable share of patients followed the intermittent 4 weeks on and 2 weeks off schedule that is the approved schedule for advanced renal cell carcinoma (RCC) and gastrointestinal stromal tumour (GIST) [26]. In addition to taking these limitations into account, it is important to highlight that any potential clinical benefits should be confirmed in studies specifically designed to evaluate whether combination therapy with a SSA is superior to the targeted agent alone. Several trials are currently ongoing: SUNLAND (ClinicalTrials.gov NCT01731925) is a clinical trial aimed at evaluating the activity of sunitinib, alone or in combination with lanreotide, in midgut carcinoids. In addition, a randomized phase II study, COOPERATE-2 (ClinicalTrials.gov NCT01374451), evaluating the treatment effect of everolimus in combination with the SSA pasireotide relative to everolimus alone on PFS in patients with advanced progressive pNET, has completed accrual. Furthermore, LUNA (ClinicalTrials.gov NCT01563354) will test the effectiveness and safety of everolimus or pasireotide alone or in combination in adult patients with advanced neuroendocrine carcinoma (typical and atypical) of the lung and thymus. The results from these studies are eagerly awaited.

Combination of lanreotide with targeted therapies did not lead to a significant increase in AEs when compared with the safety profile of each targeted agent as monotherapy. Most common AEs of SSA treatment are usually mild, limited in time, and can include local reactions 
(pain and erythema) at the injection site, abdominal cramps, nausea, flatulence, diarrhoea, steatorrhoea and a risk of cholelithiasis, more common after long exposure to the drug [2].

\section{Conclusions}

The combination of lanreotide and everolimus or sunitinib is widely used in routine clinical practice at Spanish hospitals without unexpected toxicities. The median TTP of the patients receiving the combined treatment with lanreotide appears to be clinically relevant. Furthermore, the data suggest that the combination of lanreotide and everolimus or sunitinib might provide tumour control in the majority of patients with NETs receiving treatment. The possibility of enhanced efficacy when combining SSAs and targeted therapies, suggests that this approach should be further explored in randomized prospective clinical trials.

\footnotetext{
Abbreviations

AE: Adverse event; Cl: Confidence intervals; CR: Complete response; CTCAE: Common Terminology Criteria for Adverse Events; ECOG PS: Eastern Cooperative Group Oncology Performance Status; HR: Hazard ratio; mTOR: mammalian target of rapamycin; NETs: Neuroendocrine tumours; OS: Overall survival; pNETs: Pancreatic neuroendocrine tumours; PFS: Progression-free survival; PR: Partial response; RECIST: Response Evaluation Criteria In Solid Tumours; SD: Stable disease; SSA: Somatostatin analogues; TKl: Tyrosine kinase inhibitor; TTP: Time to tumour progression; VIPoma: Vasoactive intestinal peptide secreting tumour.
}

\section{Competing interests}

The authors declare that they have no competing interests and have not received honoraria for conducting the retrospective analysis.

\section{Authors' contributions}

$J C, I S, V A, L A A$ and PGA contributed to the conception and design of the retrospective analysis. JC, IS, VA, LAA, PJF, EG, JJR, JLM, JDAL, JB, DC, JM, CL, ÁS, SC, GC, JF, JM and PGA were involved in the provision of retrospective patient data. JC, IS, VA, LAA and PGA were involved in data analysis and interpretation. $J C$ prepared the initial draft of the manuscript. All the authors actively contributed to subsequent drafts and provided final approval to submit the manuscript for publication. The corresponding author had full access to all the data and final responsibility for the decision to submit for publication. All participating investigators are listed in the acknowledgements.

\section{Acknowledgments}

We thank the participating investigators: Verónica Calderero, Hospital de Barbastro (Huesca); Juana Cano, Hospital General de Ciudad Real; Nieves Díaz, Hospital Universitario (San Juan - Alicante); Emma Dotor, Hospital Parc Taulí (Sabadell) Barcelona; María Pilar Escudero, Hospital Clínico Universitario Lozano Blesa (Zaragoza); Jose Luís Firvida, Complexo Hospitalario Universitario de Ourense; María José Gómez, Hospital Puerta del Mar (Cádiz); Encarnación Jiménez, Hospital de Jerez (Cádiz); Luís León, Hospital Clínico Universitario (Santiago de Compostela); Natalia Lupión, Hospital de Mérida (Badajoz); David Marrupe, Hospital de Móstoles (Madrid); Miguel Navarro, Hospital Clínico Universitario (Salamanca); Miguel Ruiz López de Tejada, Hospital Punta de Europa (Algeciras - Cádiz); Raquel Serrano, Hospital Reina Sofía (Córdoba); Diego Soto, Hospital Clínico Universitario (Valladolid); Alexandre Teulé, Institut Català d'Oncologia, Hospital Duran i Reynals (Barcelona); Francisca Vázquez, Hospital Clínico Universitario (Santiago de Compostela). We thank Ignasi Gich Saladich who provided support for the statistical analyses at the behest of the coordinating investigators and Aurora O'Brate who provided medical writing services subsequent to the initial draft of the manuscript, including requesting additional statistical analyses, collation of all author comments, formatting to adapt to publishing requirements, and help with submission. External commercial funding was not received for the retrospective analysis, but Ipsen Pharma, Spain provided funding for the medical writing services.

\section{Author details}

${ }^{1}$ Medical Oncology Department, Vall d'Hebron University Hospital, Autonomous University of Barcelona, P. Vall d'Hebron 119-129, 08035 Barcelona, Spain. ${ }^{2}$ Medical Oncology Department, Virgen de la Victoria University Hospital, Campus Universitario Teatinos, 29010 Málaga, Spain. ${ }^{3}$ Medical Oncology Department, Miguel Servet University Hospital, Paseo Isabel la Católica, 1-3, 50009 Zaragoza, Spain. ${ }^{4}$ Medical Oncology Department, University Hospital Complex, As Xubias, 84, 15006 A Coruña, Spain. ${ }^{5}$ Medical Oncology Department, Asturias Central University Hospital, Calle Celestino Villamil, 33006 Oviedo, Spain. ${ }^{6}$ Medical Oncology Department, Ramón y Cajal University Hospital, Ctra. de Colmenar Viejo, km. 9100, 28034 Madrid, Spain. ${ }^{7}$ Medical Oncology Department, Virgen Macarena University Hospital, Avda Dr Fedriani, 341009 Sevilla, Spain. ${ }^{8}$ Medical Oncology Department, Catalan Oncology Institute (ICO-Badalona), Germans Trias i Pujol University Hospital, Carretera Canyet s/n, Badalona, 08016 Barcelona, Spain. ${ }^{9}$ Medical Oncology Department, Virgen de la Arrixaca University Hospital, Ctra. Madrid Cartagena, 30120 Murcia, Spain. ${ }^{10}$ Medical Oncology Department, La Paz University Hospital, Paseo de la Castellana 261, 28046 Madrid, Spain. ${ }^{11}$ Medical Oncology Department, 12 de Octubre University Hospital, Avda. de Córdoba, 28041 Madrid, Spain. ${ }^{12}$ Medical Oncology Department, Toledo Hospital Complex, Av de Barber 30, 45071 Toledo, Spain. ${ }^{13}$ Medical Oncology Department, Marqués de Valdecilla University Hospital, Av Valdecilla, 39008 Santander, Spain. ${ }^{14}$ Medical Oncology Department, La Fe University Hospital, Avinguda de Campanar 21, 46026 Valencia, Spain. ${ }^{15}$ Medical Oncology Department, Cruces University Hospital, Plaza Cruces, 48903 Barakaldo, Vizcaya, Spain. ${ }^{16}$ Medical Oncology Department, Burgos University Hospital, Avda. Islas Baleares 3, 09006 Burgos, Spain. ${ }^{17}$ Medical Oncology Department, Son Dureta University Hospital, C/ Andrea Doria 55, 07014 Palma de Mallorca, Spain. ${ }^{18}$ Medical Oncology Department, Castellón Provincial Hospital Consortium, Av Doctor Clara 19, 12002 Castellón de la Plana, Spain. ${ }^{19}$ Medical Oncology Department, Gregorio Marañon Hospital, Calle Doctor Esquerdo 46, 28007 Madrid, Spain.

Received: 4 May 2014 Accepted: 23 June 2015

Published online: 04 July 2015

\section{References}

1. Lawrence B, Gustafsson BI, Chan A, Svejda B, Kidd M, Modlin IM. The epidemiology of gastroenteropancreatic neuroendocrine tumors. Endocrinol Metab Clin North Am. 2011;40(1):1-18. vii.

2. Ramage JK, Ahmed A, Ardill J, Bax N, Breen DJ, Caplin ME, et al. Guidelines for the management of gastroenteropancreatic neuroendocrine (including carcinoid) tumours (NETs). Gut. 2012;61(1):6-32.

3. Phan AT, Oberg K, Choi J, Harrison Jr LH, Hassan MM, Strosberg JR, et al, NANETS consensus guideline for the diagnosis and management of neuroendocrine tumors: well-differentiated neuroendocrine tumors of the thorax (includes lung and thymus). Pancreas. 2010;39(6):784-98.

4. Kocha W, Maroun J, Kennecke H, Law C, Metrakos P, Ouellet JF, et al, Consensus recommendations for the diagnosis and management of well-differentiated gastroenterohepatic neuroendocrine tumours: a revised statement from a Canadian National Expert Group. Curr Oncol. 2010;17(3):49-64.

5. Garcia-Carbonero R, Salazar R, Sevilla I, Isla D. SEOM clinical guidelines for the diagnosis and treatment of gastroenteropancreatic neuroendocrine tumours (GEP NETS). Clin Transl Oncol. 2011;13(8):545-51.

6. Bauer W, Briner U, Doepfner W, Haller R, Huguenin R, Marbach $P$, et al. Pless: SMS 201-995: a very potent and selective octapeptide analogue of somatostatin with prolonged action. Life Sci. 1982;31(11):1133-40.

7. Murphy WA, Lance VA, Moreau S, Moreau JP, Coy DH. Inhibition of rat prostate tumor growth by an octapeptide analog of somatostatin. Life Sci. 1987:40(26):2515-22.

8. Culler MD, Oberg K, Arnold R, Krenning EP, Sevilla I, Diaz JA. Somatostatin analogs for the treatment of neuroendocrine tumors. Cancer Metastasis Rev. 2011;30 Suppl 1:9-17.

9. Rinke A, Muller HH, Schade-Brittinger C, Klose KJ, Barth P, Wied M, et al. Placebo-controlled, double-blind, prospective, randomized study on the effect of octreotide LAR in the control of tumor growth in patients with metastatic neuroendocrine midgut tumors: a report from the PROMID Study Group. J Clin Oncol. 2009;27(28):4656-63. 
10. Sideris $L$, Dube $P$, Rinke $A$. Antitumor effects of somatostatin analogs in neuroendocrine tumors. Oncologist. 2012;17(6):747-55.

11. Massuti B, Alonso V, Mármol M, Castellano D, Fonseca E, Velasco A, et al. Evaluation of the efficacy and the safety of lanreotide on tumour growth stabilization in patients with progressive neuroendocrine tumours (NETS) who are not eligible to be treated with either surgery or chemotherapy TTD Group Study. Eur J Cancer. 2011:47 suppl 1:480-1.

12. Caplin ME, Pavel M, Ćwikła JB, Phan AT, Raderer M, Sedláčková E, et al: CLARINET Investigators. Lanreotide in metastatic enteropancreatic neuroendocrine tumors. N Engl J Med. 2014;371(3):224-33.

13. Pavel ME, Hainsworth JD, Baudin E, Peeters M, Horsch D, Winkler RE, et al. Everolimus plus octreotide long-acting repeatable for the treatment of advanced neuroendocrine tumours associated with carcinoid syndrome (RADIANT-2): a randomised, placebo-controlled, phase 3 study. Lancet. 2011;378(9808):2005-12.

14. Raymond E, Dahan L, Raoul JL, Bang YJ, Borbath I, Lombard-Bohas C, et al. Sunitinib malate for the treatment of pancreatic neuroendocrine tumors. N Engl J Med. 2011;364(6):501-13.

15. Yao JC, Shah MH, Ito T, Bohas CL, Wolin EM, Van Cutsem E, et al. Everolimus for advanced pancreatic neuroendocrine tumors. N Engl I Med. 2011;364(6):514-23.

16. Benavent M, de Miguel MJ, Garcia-Carbonero R. New targeted agents in gastroenteropancreatic neuroendocrine tumors. Target Oncol. 2012;7(2):99-106.

17. Vinik A, Van Cutsem E, Niccoli P, Raoul JL, Bang YJ, Borbath I, et al. Updated results from a phase III trial of sunitinib versus placebo in patients with progressive, unresectable, well-differentiated pancreatic neuroendocrine tumor (NET). J Clin Oncol. 2012;30:abstr 4118.

18. Bousquet C, Lasfargues C, Chalabi M, Billah SM, Susini C, Vezzosi D, et al. Clinical review: Current scientific rationale for the use of somatostatin analogs and mTOR inhibitors in neuroendocrine tumor therapy. J Clin Endocrinol Metab. 2012;97(3):727-37.

19. Yao JC, Lombard-Bohas C, Baudin E, Kvols LK, Rougier P, Ruszniewski P, et al. Daily oral everolimus activity in patients with metastatic pancreatic neuroendocrine tumors after failure of cytotoxic chemotherapy: a phase II trial. J Clin Oncol. 2010;28(1):69-76.

20. Yao JC, Phan AT, Chang DZ, Wolff RA, Hess K, Gupta S, et al. Efficacy of RAD001 (everolimus) and octreotide LAR in advanced low- to intermediategrade neuroendocrine tumors: results of a phase II study. J Clin Oncol. 2008;26(26):4311-8.

21. Fonseca PJ, Uriol E, Galván JA, Álvarez C, Pérez Q, Villanueva N, et al. Prolonged clinical benefit of everolimus therapy in the management of high-grade pancreatic neuroendocrine carcinoma. Case Rep Oncol. 2013;6(2):441-9.

22. Castellano D, Grande E, Barriuso J. Advances in pancreatic neuroendocrine tumor treatment. N Engl J Med. 2011;364(19):1872-3. author reply 1873-1874.

23. Barriuso J, Grande E, Quindós Varela M, Sereno M, López C, Sepulveda J, et al. Sunitinib efficacy and tolerability in patients with neuroendocrine tumors out of a trial: a Spanish Multicenter Cohort. Ann Oncol. 2010;21 suppl 8:viii264 abstr 847P.

24. Shah MH, Lombard-Bohas C, Ito T, Wolin EM, Van Cutsem E, Sachs C, et al. Everolimus in patients with advanced pancreatic neuroendocrine tumors (pNET): Updated results of a randomized, double-blind, placebo-controlled, multicenter phase III trial (RADIANT-3). J Clin Oncol. 2011;29(suppl):abstr 4010.

25. Valle J, Faivre S, Raoul J, Bang Y, Patyna S, Lu DR, et al. Phase III trial of sunitinib versus placebo for treatment of pancreatic neuroendocrine tumors: impact of somatostatin analogue treatment on progression-free survival. Ann Oncol. 2010;21 suppl 8:viii264 abstr 846P.

26. Raymond E, Faivre S. Learning experiences with sunitinib continuous daily dosing in patients with pancreatic neuroendocrine tumours. Curr Oncol. 2014;21(6):309-17.

\section{Submit your next manuscript to BioMed Central and take full advantage of:}

- Convenient online submission

- Thorough peer review

- No space constraints or color figure charges

- Immediate publication on acceptance

- Inclusion in PubMed, CAS, Scopus and Google Scholar

- Research which is freely available for redistribution 